\title{
A giant virus genome is densely packaged by stable nucleosomes
}

Terri D. Bryson ${ }^{1,2} \uparrow$, Pablo De Ioannes ${ }^{3} \uparrow$, Marco Igor Valencia-Sánchez ${ }^{3}$, Jorja G. Henikoff ${ }^{1}$, Paul B. Talbert $^{1,2}$, Bernard La Scola ${ }^{4,5}$, Karim-Jean Armache ${ }^{3 *}$, Steven Henikoff ${ }^{1,2 *}$

${ }^{1}$ Basic Sciences Division, Fred Hutchinson Cancer Research Center; Seattle, WA, 98109, USA

${ }^{2}$ Howard Hughes Medical Institute; Chevy Chase, MD, USA

${ }^{3}$ Skirball Institute of Biomolecular Medicine, Department of Biochemistry and Molecular Pharmacology, New York University Grossman School of Medicine, New York, NY, USA

${ }^{4}$ Institut de Recherche Pour le Développement (IRD), Assistance Publique - Hôpitaux de Marseille (APHM), MEPHI, Aix-Marseille University, Marseille, France.

${ }^{5}$ IHU Méditerranée Infection, Marseille, France

$\dagger$ Equal contributions

*Co-corresponding authors. Email: karim-jean.armache@nyulangone.org, steveh@,fredhutch.org

\begin{abstract}
The doublet histones of Marseillevirus are distantly related to the four eukaryotic core histones and wrap DNA to form remarkably similar nucleosomes. By releasing Marseillevirus chromatin from virions into solution and performing genome-wide nuclease digestion and chemical cleavage assays, we find that the higher-order organization of Marseillevirus chromatin differs greatly from that of eukaryotes.

Marseillevirus nucleosomes fully protect DNA within virions, without linker DNA or phasing along genes. Likewise, we observe that most nucleosomes reconstituted onto 3-copy tandem repeats of a nucleosome positioning sequence are tightly packed and fully wrapped. We also document repeat generation and instability during viral passage in amoeboid culture. Dense promiscuous packing of fully wrapped nucleosomes rather than 'beads-on-a-string' with genic punctuation suggests a viral genome protection function for doublet histones.
\end{abstract}


First isolated in 2007, the giant Marseillevirus encapsulates a 368-kb circular genome encoding 457 proteins (1), and related viruses of the family Marseilleviridae have since been discovered infecting Acanthamoeba species worldwide (2). Marseillevirus genomes encode doublet histones that are paired homologs of the four eukaryote core histones, where $\mathrm{H} \beta-\mathrm{H} \alpha$ is homologous to eukaryotic $\mathrm{H} 2 \mathrm{~B}$ and $\mathrm{H} 2 \mathrm{~A}$ and $\mathrm{H} \delta-\mathrm{H} \gamma$ is homologous to $\mathrm{H} 4$ and $\mathrm{H} 3$. Phylogenetic analysis places $\mathrm{H} \alpha, \mathrm{H} \beta, \mathrm{H} \gamma, \mathrm{H} \delta$ as sister groups respectively to their $\mathrm{H} 2 \mathrm{~A}, \mathrm{H} 2 \mathrm{~B}, \mathrm{H} 3$ and $\mathrm{H} 4$ eukaryotic counterparts, consistent with divergence within the Marseilleviridae from the last eukaryotic common ancestor for all four histone folds (3). We and others have previously used biochemical reconstitution and cryoEM imaging to solve the high-resolution structure of Marseillevirus nucleosomes, which show a striking resemblance to their eukaryotic counterparts $(4,5)$. Unlike octameric eukaryotic nucleosomes, which wrap 147 bp of DNA, tetrameric Marseillevirus nucleosomes wrap only $121 \mathrm{bp}$ of DNA, despite being reconstituted on the Widom 601 artificial positioning sequence, selected for 147-bp wrapping of nucleosome cores.

Because Marseillevirus doublets assemble into nucleosomes that are not fully wrapped by DNA, it was assumed that they are inherently unstable, perhaps to facilitate expression during early stages of infection or for gene regulation (5). However, without isolation of viral chromatin in its native form, the extent to which reconstituted Marseillevirus nucleosomes are representative of their conformation in virions is debatable $(6,7)$. By mapping the chromatin landscape of the viral genome we aimed to understand the functional and evolutionary basis for viral nucleosomal packaging.

The capsids of giant viruses that infect amoeba are resistant to treatments that disrupt other viruses, however by dialyzing in low $\mathrm{pH}$ conditions Schrad and co-workers (8) have shown that giant Mimivirus capsids can be breached, although without releasing their contents. We followed their protocol to open the Marseillevirus capsid, then dialyzed into a neutral buffer for Micrococcal Nuclease (MNase) digestion. High yields of digested chromatin were obtained from samples treated with $0.5 \%$ NP-40 over a time-course ranging from 1 minute to 18 hours (fig. S1). In contrast, 0.1\% digitonin had no effect. As NP-40 permeabilizes membranes by sequestering lipids, whereas digitonin permeabilizes cell membranes by displacing membrane sterols, our results suggest that the chromatin of Marseilleviridae, like that of giant Mimiviridae (9-12), is enclosed within an impermeable lipid membrane that would have protected it from low $\mathrm{pH}$ conditions and then permeabilized for MNase to gain access. 
bioRxiv preprint doi: https://doi.org/10.1101/2022.01.15.476465; this version posted January 16,2022 . The copyright holder for this preprint (which was not certified by peer review) is the author/funder, who has granted bioRxiv a license to display the preprint in perpetuity. It is made available under aCC-BY 4.0 International license.

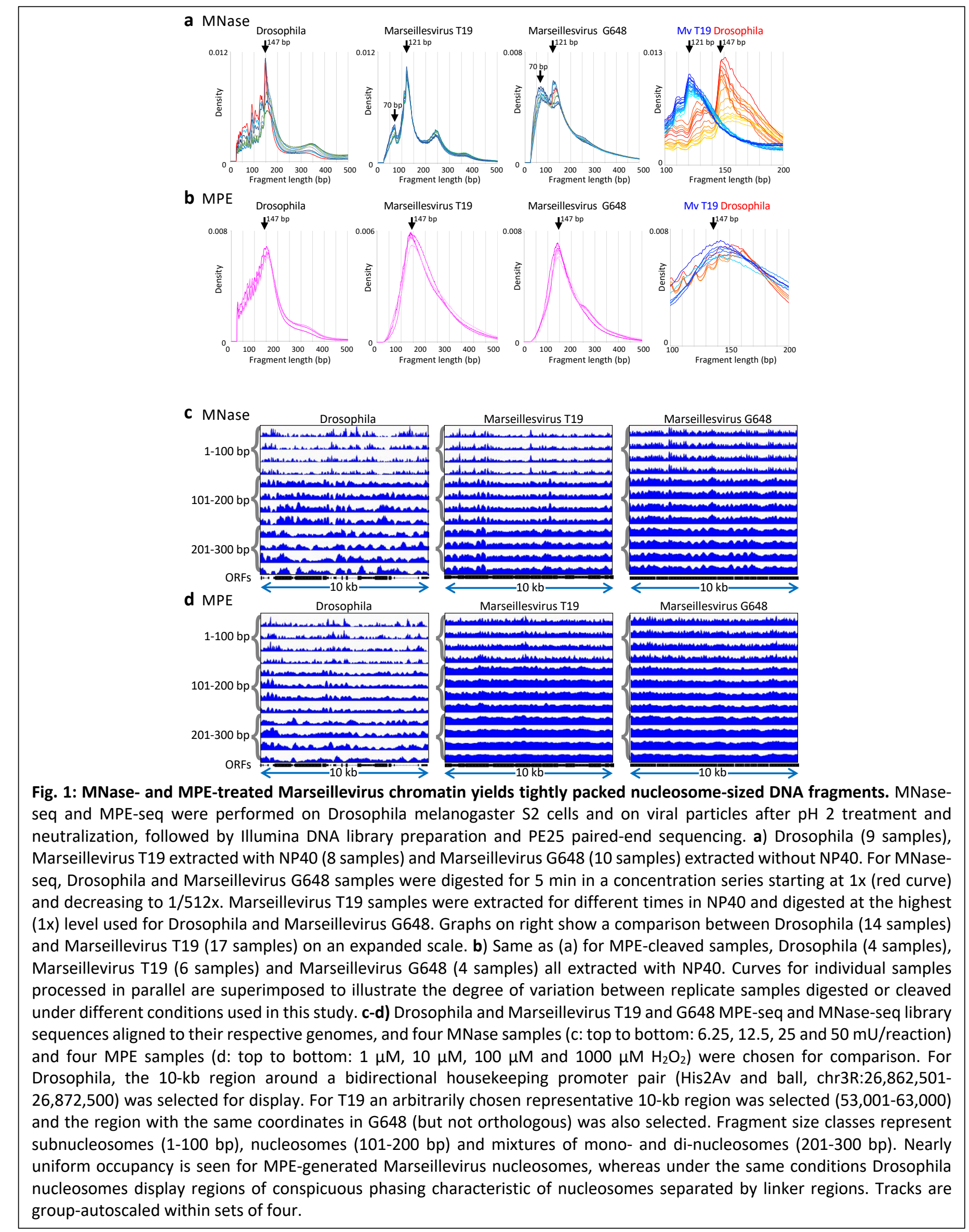


Under our permeabilization and digestion conditions, $85-90 \%$ of the DNA in virions was recovered as intact MNase-protected particles. At the highest MNase digestion levels, control Drosophila nucleosomes showed a dominant 147-bp peak, but also a smear of sub-nucleosome-sized digestion products (Fig. 1a). Using the highest digestion level for Marseillevirus we observed a dominant 121-bp MNase-protected peak, a small 70-bp peak, but no smear, confirming cryoEM observations of 121-bp wrapping of reconstituted nucleosome cores $(4,5)$. These observations indicate that Marseillevirus nucleosomes are less sensitive to intranucleosomal cleavages than are eukaryotic nucleosomes.

MNase is an endo/exo-nuclease that is known to preferentially digest AT-rich regions $(13,14)$. Consistent with these observations, we find that $\sim 90 \%$ of the cleavage sites occur between A/T base pairs (fig. S2) and long AT-rich regions are preferentially digested (fig. S3). Therefore, we wondered whether the unexpected $\sim 70$ bp peak (Fig. 1a) resulted from aggressive MNase digestion of AT-rich regions within nucleosomes. To eliminate this potential artifact, we used a small-molecule DNA cleavage reagent, methidiumpropylEDTA-Fe(II) (MPE) which hydrolyzes DNA phosphodiester bonds, where $\mathrm{H}_{2} \mathrm{O}_{2}$ provides reactive oxygen for MPE-Fe(II)-catalyzed DNA cleavage (15). MPE-seq is performed similarly to MNase-seq, but without exonuclease activity and without sequence bias (16) (fig. S3). When we treated Drosophila nuclei and permeabilized Marseillevirus particles with MPE-Fe(II), we observed mostly mononucleosome-sized particles for Drosophila, with a 150 -bp peak and periodic 10-bp internal cleavages (Fig. 1b). MPE-seq of Marseillevirus chromatin from both the original isolate (strain T19, later named Marseillevirus marseillevirus) and a more recent isolate, G648 (1), also revealed a 150-bp peak with close concordance between samples, and no 70-bp peak. The lack of periodic 10-bp internal cleavages demonstrates that Marseillevirus chromatin is highly refractory to intranucleosomal cleavages (Fig. 1b). Whereas MNase digestion resulted in a 26-bp fragment size difference between Marseillevirus and Drosophila nucleosomes (Fig. 1a right panel), MPE cleavage resulted in fragments of nearly the same size (Fig. 1b, right panel). These consistent discrepancies between MNase- and MPE-generated fragments indicate that the aggressive endo/exonucleolytic activity of MNase and its preference for AT-rich DNA is responsible for the $\sim 70$-bp fragment peaks in T19 and G648 (fig. S2). Although the genomes of both Marseillevirus isolates have the same $\sim 44 \%$ GC-content as Drosophila, they have characteristic A/T homopolymeric runs in promoter regions (17) that might contribute to artifactual internal MNase cleavages seen for Marseillevirus nucleosomes.

Interestingly, Marseillevirus nucleosomes remain insoluble even after MNase digestion, in contrast to Drosophila nucleosomes, which are released by MNase into solution (fig. S4), as if tight packaging within 
the permeabilized virion prevents release of mono-nucleosomes. To examine the global organization of Marseillevirus nucleosomes, we separated fragments by size in 100-bp intervals and displayed a representative 10-kb region of the T19 and G648 genomes for MNase- and MPE-generated replicate samples. Typical Drosophila chromatin profiles showed variable nucleosome positioning with patches of positioned nucleosomes for 101-200 bp fragments using either MPE cleavage or MNase digestion, but not for subnucleosomal 1-100 bp or supranucleosomal 201-300 bp fragments (Fig. 1c-d). In contrast, the 101200 bp MPE-generated fragments from both T19 and G648 nucleosomes showed at most minor variations that were consistent between replicates over a uniform chromatin landscape with no conspicuous positioning in the 101-201 bp subset (Fig. 1d). This lack of positioning in Marseillevirus T19 and G648 nucleosomes suggests that they are tightly packed without intervening linkers characteristic of eukaryotic nucleosomes.

We wondered whether the minor regularities seen in the MNase-digested Marseillevirus nucleosome landscapes correspond to genic regions, which are annotated as Open Reading Frames (ORFs) and are separated by very short spans that are usually AT-rich (17). To investigate this possibility, we aligned all 191 Open Reading Frames (ORFs) that are $\geq 600$ bp at their 5' ends and averaged each nucleotide position over gene bodies. For comparison we chose the first 191 ORFs $\geq 600 \mathrm{bp}$ from the chronological list of Drosophila ORFs. Alignment of MNase-generated 101-200 bp fragments to the 191 Drosophila ORFs revealed the characteristic translational phasing pattern, with a prominent +1 nucleosomal peak just downstream of the ORF 5 ' end and phased +2 and +3 nucleosomes with reduced occupancy for all digestion levels over a 128-fold range (Fig. 2a). MPE-generated fragments showed the same 5'-aligned translational phasing patterns for 101-200 bp fragments, confirming that maps produced using MPE and MNase are approximately concordant for control eukaryotic nucleosomes. In contrast, MNase-generated Marseillevirus T19 and G648 101-200 bp fragments displayed very little if any 5' phasing when plotted on the same scale (Fig. 2a, c), although when the scale was expanded a phased +1 nucleosome was observed. However, no phasing was seen for MPE-generated T19 and G648 5'-aligned ORFs, indicating that the minor phasing that was seen with MNase is attributable to internal cleavages within nucleosomes. The lack of 5' phasing, a universal characteristic of eukaryotic genes, indicates that the packaged Marseillevirus genome is inactive. Alignment of the 191 ORFs at their 3' ends shows extreme sensitivity of Drosophila nucleosomes to MNase levels not seen for MPE-generated fragments (Fig. 2b), which is consistent with partial unwrapping of AT-rich ORF 3'-end DNA from nucleosome cores and sensitivity to MNase exonucleolytic activity. A similar MNase sensitivity and MPE insensitivity is seen for T19 and G648 


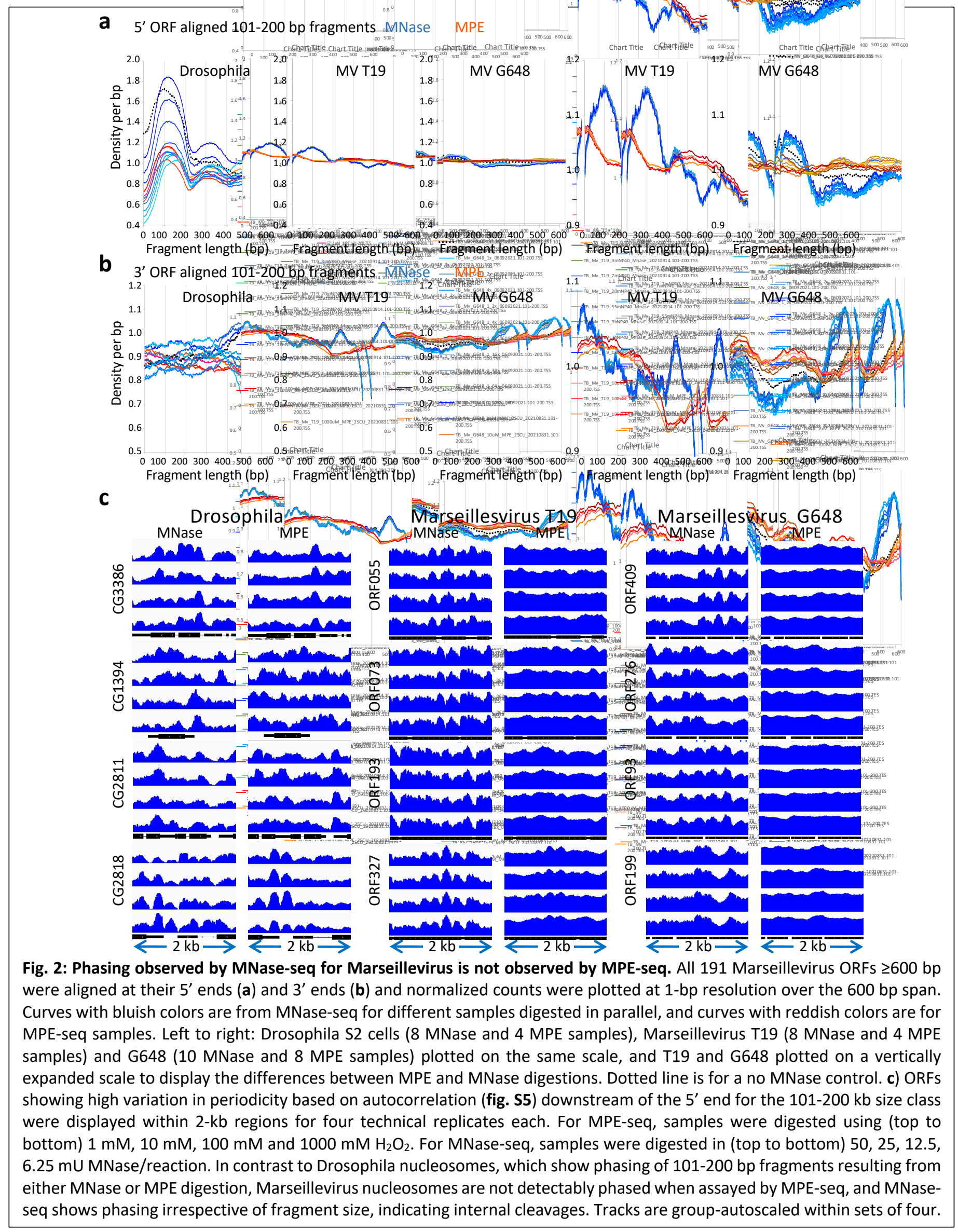


nucleosomes at the very 3' ends of ORFs (Fig. 2b), which is likewise attributable to AT-rich regions at Marseillevirus 3' ends. However, unlike chromatin at the 3' ends of Drosophila ORFs, both T19 and G648 chromatin displayed an average peak of MNase resistance just upstream of the 3' end. As this peak was absent from MPE-digested average profiles, we attribute it to internal MNase cleavage of a subset of nucleosomes that are relatively excluded from neighboring AT-rich regions rather than to 3' nucleosome phasing. The lack of any chromatin accessibility features punctuating genic regions implies that Marseillesvirus nucleosomes have evolved exclusively for packaging within the virion.

Mapping of Marseillevirus fragments to the T19 genome assembly revealed an unexpected overrepresentation of fragments relative to the rest of the genome in two sharply defined regions (Fig. 3a, left panel). A 20-kb region centered over Position 27,000 is $\sim 3$-fold over-represented and an $\sim 2-\mathrm{kb}$ region centered over Position 318,400 is $\sim 10$-fold over-represented similarly in all ten MNase datasets regardless of digestion level and in all four MPE datasets regardless of $\mathrm{H}_{2} \mathrm{O}_{2}$ concentration. To determine whether these striking over-representation features were present in the initial sample from 2007 used to assemble the original T19 map, we mapped the primary T19 reads from the original fastq files, and observed that over-representation at Position 27,000 was already conspicuously present in this sample (Fig. 3a, top track). To quantify over-representation, we randomly sampled fragments of similar lengths to each overrepresented feature from the rest of the genome 1000 times and plotted the abundance of each sample on a $\log$-log cumulative plot for the raw reads from the original 2007 virus culture and the No-MNase pairedend reads from the 2020 virus culture used in this study (Fig. 3b, left panel). This revealed that the 20-kb region over Position 27,000 was $\sim 2$-fold over-represented in the original 2007 viral culture and $\sim 2.7$-fold over-represented in the 2020 culture relative to their respective genome-wide median abundances.

Likewise, the 2-kb region over Position 318,400 was $\sim 1$.1-fold over-represented in the original 2007 viral culture and $\sim 10$-fold over-represented in the 2020 culture. Although the magnitude of over-representation of the 2-kb region in the original 2007 viral culture is small, it is ranked between \#1 and \#2 of the 1000 randomly sampled regions, and so likely represents incipient over-representation of some virions in that culture. It is evident that over-representation of the $20-\mathrm{kb}$ region continued to be maintained at 2-3-fold over-abundance during successive passages in Acanthamoeba polyphaga culture at similar levels within the cultured Marseillevirus populations, while the $2-\mathrm{kb}$ region expanded $\sim 12$-fold during successive passages in culture. 


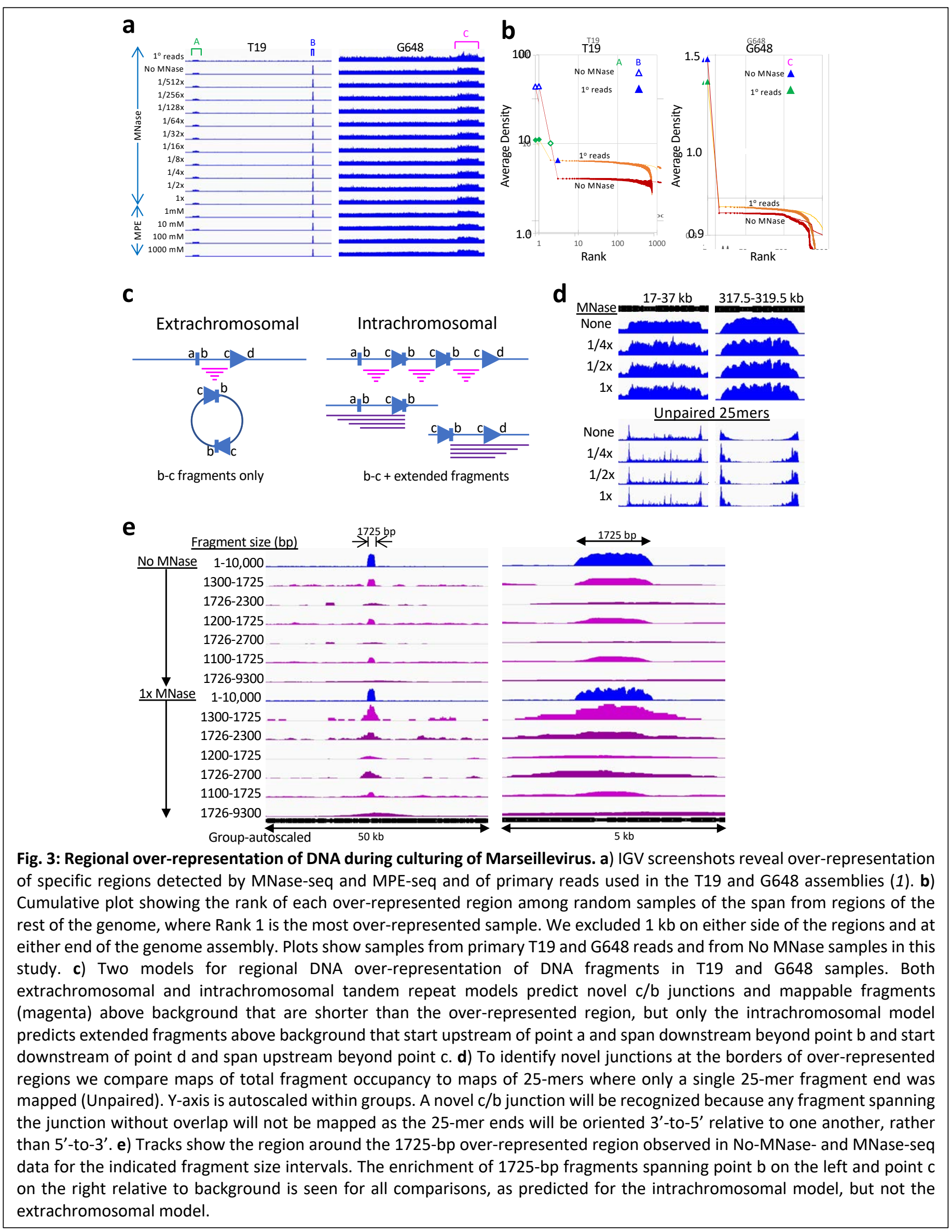


We next asked if regional over-representation and expansion during culturing is a general feature of the Marseilleviridae (1). G648 is a much more recent isolate and therefore has been maintained for a shorter time in culture than T19. For G648 we observed a different region of over-representation, in which a $\sim 50$ kb segment approximately centered over Position 319,000 is present in all ten samples in the MNase concentration series and identical results were obtained using MPE digestion (Fig. 3a, right panel). Although the primary reads from the fastq files used for the original assembly of G648 were relatively sparse, quantitative sampling analysis showed that this $\sim 50-\mathrm{kb}$ region also strongly over-represented, with a 1.9-fold excess, compared to a 2.6-fold excess for the No MNase sample (Fig. 3b right panel). We conclude that sporadic regional over-representation is a general feature of Marseillevirus culturing in $A$. polyphaga, even for newly isolated virions. Analysis of long repeat-spanning fragments and unpaired 25 mer fragment ends indicated that regional over-representation is accounted for by intrachromosomal tandem repeats rather than extrachromosomal circles (Fig. 3c-e).

We wondered whether previous reconstitutions of Marseillevirus nucleosomes on single 601 sequences (4, 5) failed to show fuller wrapping because of the lack of neighboring nucleosomes, which are closely abutted in virions. Accordingly, we performed MPE and MNase digestion on native and cross-linked reconstituted Marseillevirus chromatin that had been assembled at $60 \mu \mathrm{g} / \mathrm{ml}$ or $300 \mu \mathrm{g} / \mathrm{ml}$ concentrations onto a three-copy 601 array and onto a 12-copy 601 array. Following DNA extraction, we prepared sequencing libraries and performed paired-end sequencing, aligning fragments from native or cross-linked 3-copy and 12-copy chromatin digests to the 3-copy 601 array. For the 3-copy native chromatin, both MPE and MNase showed similar chromatin landscapes for the $60 \mu \mathrm{g} / \mathrm{ml}$ and $300 \mu \mathrm{g} / \mathrm{ml} \mathrm{samples,} \mathrm{with} \mathrm{higher}$ occupancy over the three 147-bp 601 positioning sequences than over the intervening 40-bp linkers (Fig. 4a). Although the net occupancy over positioning sequence and linker differed by less than half, consistent with high occupancy throughout the array, the transitions between 601 and linker sequence were sharply defined, indicating an excess of 147-bp 601 particles precisely positioned over the 601 sequence. A much smaller excess of 147-bp 601 particles was observed for MPE digestion of cross-linked 3-copy chromatin.

To quantify the relative abundance of precise 147-bp 601 particles, we plotted the length distributions for each 3-copy sample (Fig. 4b). In each case, MPE digestions of 3-copy array chromatin resulted in 1-bp wide fragment length peaks at $147 \mathrm{bp}, 334 \mathrm{bp}$ and $521 \mathrm{bp}$. As the entire 3-copy array is $521 \mathrm{bp}$, and $334 \mathrm{bp}$ is precisely the size expected for a 601-linker-601 spanning fragment, it is evident that MPE endonucleolytically digests to completion without detectable encroachment into the nucleosome-wrapped 


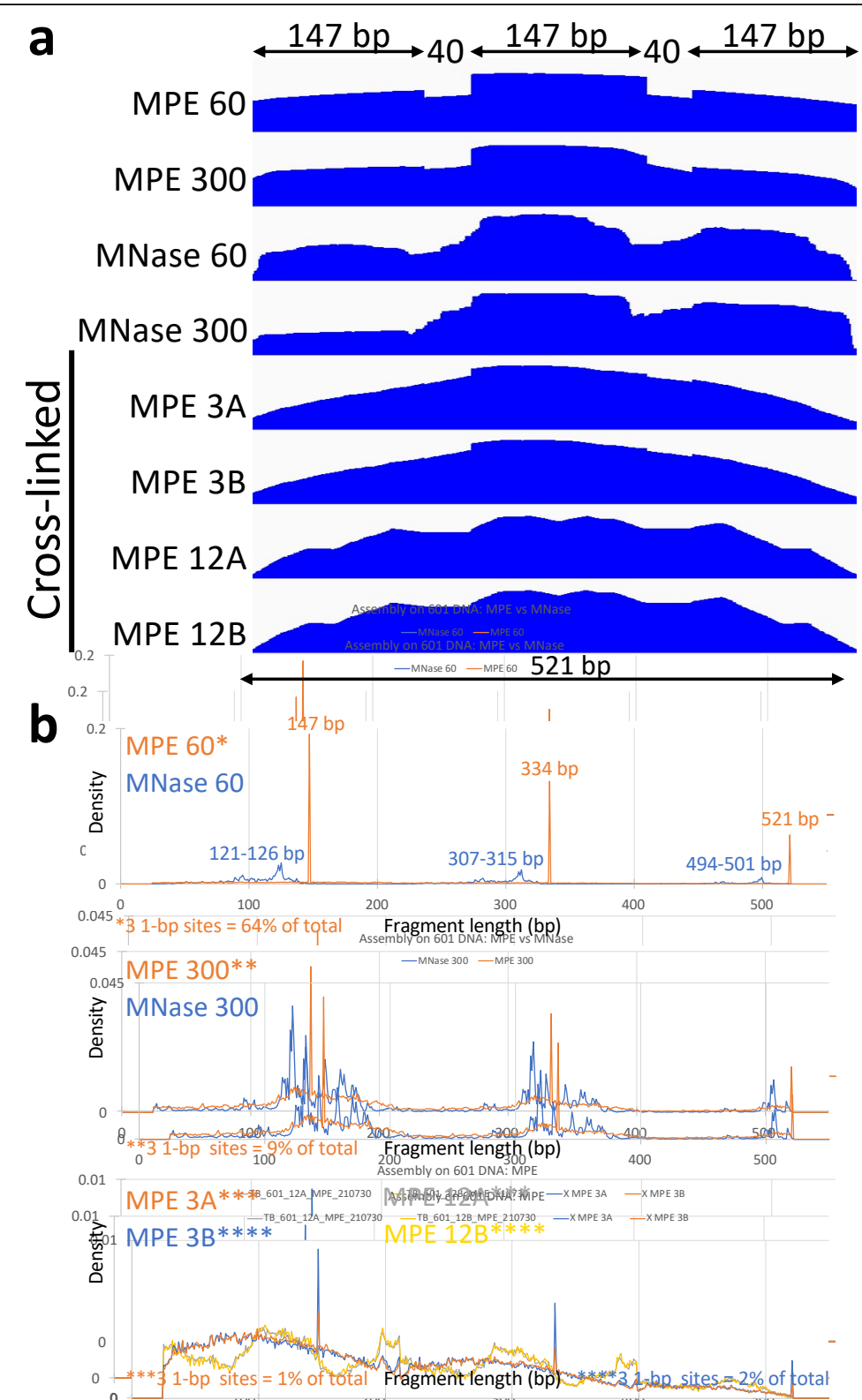

Fig. 4: Assembly of Marseillevirus histones onto Widom 601 arrays produces 147-bp nucleosomes. Three-copy or 12-copy Widom 601 arrays and $60 \mu \mathrm{g} / \mathrm{ml}$ or $300 \mu \mathrm{g} / \mathrm{ml}$ of Marseillevirus histones were assembled into nucleosomes and subjected to MPE-seq and MNase-seq. a) Profiles across the 521-bp 3-copy array map were group autoscaled. The higher abundance of fragments towards the middle of each array is a consequence of mapping to a linear fragment, where fragments that span an end can only align with internal copies. MPE3A and MPE3B were assembled onto a 521-bp 3-copy array and MPE12A and MPE12B were assembled onto a 2084-bp 12-copy Widom 601 array, cross-linked, digested with MPE and aligned to the 3-copy array map. b) Length distributions of fragments produced from the 3-copy array reconstituted samples show that $\sim 40 \%$ of the total occupancy is accounted for by precise cleavages on either or both ends of a 147-bp particle.

particle. Many particles in the $60 \mu \mathrm{g} / \mathrm{ml}$ sample were precisely phased over the 601 positioning sequence, as $147 \mathrm{bp}, 334 \mathrm{bp}$ and $521 \mathrm{bp}$ fragments accounted for $\sim 40 \%$ of the total assembled chromatin. One basepair wide peaks at these positions in the plot were also seen for cross-linked 3-copy Marseillevirus chromatin arrays, superimposed over a broad distribution of fragment lengths indicative of densely packed chromatin. In contrast to the results with MPE, MNase digestion of the same reconstituted chromatin 
showed a distribution of fragment lengths $\sim 20-25$ bp smaller than the three discrete fragment lengths produced by MPE digestion. This difference in fragment length for reconstituted Marseillevirus chromatin is similar to the difference observed for digestion of viral chromatin, and provides confirmation that assembly onto a tandem array of positioning sequences as opposed to assembly onto a single positioning sequence recapitulates the situation in virio. Cross-linking resulted in reduced precision of MPE digestion for 3-copy array chromatin and total absence of 1-bp peaks for the cross-linked 12-copy array, which suggests that inter-nucleosomal cross-links of closely abutted nucleosomes interfered with the penetration or cleavage activity of MPE.

In summary, we have shown that Marseillevirus nucleosomes can be recovered intact from virions and used to elucidate nucleosome organizational features and document genomic instability using both MNase-seq and MPE-seq. These methods reveal particles that differ from eukaryotic nucleosomes in being refractory to internal cleavages and tightly packed into a landscape without linker DNA or phasing around genes. MPE-seq also shows that the nucleosomes of two Marseillevirus isolates protect $\sim 147$ bp of DNA, confirmed by the observation of fully wrapped 147-bp reconstituted particles on Widom 601 DNA. Taken together, our findings reveal a dense chromatin landscape that may have evolved to maximize protection of viral DNA for survival during infection in amoeba cytoplasm. Following infection, the tightly packed undifferentiated Marseillevirus chromatinized genome, surrounded by a lipid membrane (9-12), becomes incorporated into a "viral factory" where it undergoes cycles of replication and gene expression within Acanthamoeba cytoplasm, cytologically separate from the cell nucleus $(5,12)$. This separation may have facilitated viral histones specializing for chromatin condensation rather than gene regulation.

\section{Materials and Methods}

\section{Viruses}

Marseillevirus marseillevirus Strains T19 (2) and G648 (1) were cultured in Acanthamoeba polyphaga as described (18).

\section{Drosophila cells}

Drosophila S2 cells were grown in HyClone SFX Insect Cell Culture Media (Cytiva SH30278.02) supplemented with $18 \mathrm{mM}$ L-Glutamine, seeded at $2 \times 10^{6} / \mathrm{mL}$ three times per week, and harvested with $>95 \%$ viability at mid-log phase. A total of $1 \times 10^{7}$ cells were centrifuged in a swinging-bucket rotor for 4 min at $700 \mathrm{xg}$ at $25^{\circ} \mathrm{C}$ and washed twice in cold $1 \mathrm{x}$ PBS. The cell pellet was resuspended in $1 \mathrm{~mL}$ TM2+PI 
(10mM Tris $\mathrm{pH} 8,2 \mathrm{mM} \mathrm{MgCl}_{2}+$ Protease Inhibitor, Sigma 11836170001) and chilled in ice water for 1 min. NP-40 was added to $0.5 \%$ and vortexed gently at half maximum speed for $\sim 3 \mathrm{sec}$ and returned to ice water slurry. Release of nuclei was ascertained by microscopic observation of aliquots until $\geq 80 \%$ of cellular membranes were disrupted $(\sim 3 \mathrm{~min})$. Nuclei were centrifuged $10 \mathrm{~min}$ at $150 \mathrm{xg}$ at $4^{\circ} \mathrm{C}$, washed twice in $1.5 \mathrm{~mL}$ TM2+PI and finally resuspended in $200 \mu \mathrm{L}$ TM2+PI. Each digestion reaction contained either $50 \mathrm{~K}$ or $150 \mathrm{~K}$ nuclei per timepoint.

\section{Viral capsid opening and permeabilization}

We followed the viral opening procedure described by Schrad et al., 2020 (8) for Mimivirus, with minor modifications. Each sample from a purified Marseillevirus culture was applied to the membrane of a $7 \mathrm{~K}$ MWCO Dialysis Unit (Thermo 69562) and dialyzed against $250 \mathrm{~mL}$ of $20 \mathrm{mM}$ sodium phosphate buffer adjusted to $\mathrm{pH} 2,2 \mathrm{mM} \mathrm{MgCl}, 1 \mathrm{mM}$ PMSF for $2 \mathrm{hr}$ at $25^{\circ} \mathrm{C}$, followed by dialysis against $250 \mathrm{~mL} \mathrm{TM} 2,1$ mM PMSF for $3 \mathrm{hr}$. In some experiments viral suspensions were diluted with TM2 prior to dialysis. To equalize the amounts of DNA from Marseillevirus and Drosophila control cells, DNA was extracted from a range of volumes of purified Marseillevirus culture in parallel with a known number of S2 cells. Either 0.5 $\mu \mathrm{L}$ or $1.5 \mu \mathrm{L}$ of Marseillevirus culture was used per timepoint.

To improve recovery, we included the non-ionic detergent NP-40, which is widely used for chromatin release from cells. Recovery from Marseillevirus T19 particles was $\sim 5-6 \%$, but increased to $\sim 85-90 \%$ in $0.5 \%$ NP-40 using the maximum MNase digestion conditions that had resulted in mostly mononucleosomes in the previous Drosophila experiments (fig. S1c-e).

\section{Nucleosome reconstitution}

Expression and purification of Marseillevirus histones, purification of Widom 601 DNA arrays and nucleosome assembly was performed as previously described (4).

\section{MNase-seq}

MNase-seq was performed as previously described (19). Briefly, Micrococcal Nuclease (MNase, Sigma $\mathrm{N} 3755$ ) was reconstituted to a concentration of 1 unit per $5 \mu 1$ with nuclease-free water, aliquoted and stored at $-20^{\circ} \mathrm{C}$. MNase was thawed on ice and diluted stepwise 1:1 from 1x to $1 / 512 \mathrm{x}$ with TM2 buffer (10 $\mathrm{mM}$ Tris $\mathrm{pH} 8,2 \mathrm{mM} \mathrm{MgCl} 2$ ) where $1 \mathrm{x}$ is $20 \mathrm{mU} / \mu \mathrm{L}$. The volume of each Drosophila or Marseillevirus timepoint was adjusted to $166 \mu \mathrm{L}$ with TM2+PI (Protease Inhibitor, Sigma 11836170001), and $2.5 \mu \mathrm{L}$ of each MNase dilution was added with mixing, then heated to $37^{\circ} \mathrm{C}$ for $1 \mathrm{~min}$. MNase was activated by 
addition of $3.5 \mu \mathrm{L}$ of $100 \mathrm{mM} \mathrm{CaCl}_{2}$ and incubated for $5 \mathrm{~min}$ at $37^{\circ} \mathrm{C}$. Reactions were stopped with $172 \mu \mathrm{L}$ of 2xSTOP solution (10mM Tris, $2 \mathrm{mM} \mathrm{MgCl}_{2}, 340 \mathrm{mM} \mathrm{NaCl}, 20 \mathrm{mM}$ EDTA, 4mM EGTA, 100ug/mL RNase, DNase-free (Sigma11119915001) and DNA was extracted as described below. For reconstituted nucleosomes, we digested with $0.2 \mathrm{U}$ MNase/ $\mu \mathrm{g}$ DNA for $7 \mathrm{~min}$ at room temperature (20).

We performed digestions over a time course and concentration range that we had previously found to be sufficient for digesting chromatin from Drosophila S2 cells into mono- and oligo-nucleosomes (19). In that study, 1 minute digestions at $2.5 \mathrm{U} /$ million cells had yielded an electrophoretic 'ladder' dominated by oligonucleosomes, 5 minute digestions yielded mostly mononucleosomes, and 30 minute digestions resulted in partially degraded mononucleosomes (30 min). By Tapestation analysis we observed a Marseillevirus nucleosomal ladder, with slightly smaller mononucleosome fragment sizes and much shorter distances between successive peaks than observed for Drosophila digested under same conditions (fig. S2b).

We prepared MNase-seq Illumina sequencing libraries from the resulting MNase-digested fragments for both NP-40-treated and untreated libraries, performed paired-end DNA sequencing, and mapped the resulting read pairs to the annotated Marseillevirus T19 genome assembly. On average 97\% of fragments mapped to this assembly, confirming the purity of our viral sample. Fragment lengths inferred from sequencing data are smaller than what we observed by Tapestation analysis of purified DNA following MNase digestion (fig. S1d), which reflects the selection for smaller fragments during PCR.

\section{MPE-seq}

MPE-seq was performed as described by Ishii et al. (16). Briefly, the opened Marseillevirus and S2 nuclei were treated with hydrogen peroxide across the range of $0.001-1 \mathrm{mM}$ and cleavage was induced by addition of methidiumpropyl-EDTA-Fe(II) (MPE, a generous gift from Jim Kadonaga), which is MPE complexed with ammonium iron(II) sulfate) at $10 \mu \mathrm{M}$ or $40 \mu \mathrm{M}$ for $5 \mathrm{~min}$ at room temperature. The reaction was quenched with $6 \mathrm{mM}$ bathophenanthroline (Sigma 133159), followed by 2xSTOP buffer (10 mM Tris, 2 $\mathrm{mM} \mathrm{MgCl}$, $340 \mathrm{mM} \mathrm{NaCl}, 20 \mathrm{mM}$ EDTA, 4 mM EGTA, $100 \mu \mathrm{g} / \mathrm{mL}$ RNase, DNase-free) at a volume equal to the sample and total DNA extracted as described below. For reconstituted nucleosomes, we digested with $40 \mu \mathrm{M}$ MPE using at a ratio of $1 \mathrm{mM} \mathrm{H}_{2} \mathrm{O}_{2} / 650 \mathrm{ng}$ DNA for $5 \mathrm{~min}$ at room temperature.

\section{DNA extraction}


Sample volumes were adjusted to $\sim 340 \mu \mathrm{L}$ with TM2. To each sample, $3.4 \mu \mathrm{L} 10 \%$ SDS and $2.5 \mu \mathrm{L}$ Proteinase K $(20 \mathrm{mg} / \mathrm{ml})$ were added and incubated $30 \mathrm{~min} 55^{\circ} \mathrm{C}$. DNA was extracted once with $350 \mu \mathrm{L}$ Phenol:Chloroform:Isoamyl Alcohol (25:24:1) in a phase-lock tube (Qiagen cat. no. 129046) and centrifuged $5 \mathrm{~min}$ at $16,000 \mathrm{xg}$ followed by extraction with $350 \mu \mathrm{L}$ chloroform. The aqueous layer was transferred to a fresh tube containing $2 \mu \mathrm{L}$ Glycogen $(20 \mathrm{mg} / \mathrm{mL}$ Sigma cat. no. 10901393001$)$. DNA was precipitated with three volumes of $100 \%$ ethanol and centrifuged at $16,000 \mathrm{xg}$ for $30 \mathrm{~min}$ at $4^{\circ} \mathrm{C}$.. The pellet was rinsed twice with $1 \mathrm{ml} \mathrm{80 \%}$ ethanol, air dried and dissolved in $30 \mathrm{uL} 10 \mathrm{mM}$ Tris $\mathrm{pH}$ 8.0. Two $\mu \mathrm{L}$ was analyzed using a HDS1000 ScreenTape on an Agilent 4200 TapeStation.

\section{Library Preparation}

Sequencing libraries were prepared from DNA fragments using the KAPA HyperPrep Kit (KAPA cat. no. KK8504) following the manufacturer's instructions. Libraries were amplified for eight cycles using a 10 $\sec 60^{\circ} \mathrm{C}$ combined annealing/extension step. Alternatively, a $72^{\circ} \mathrm{C} 1 \mathrm{~min}$ PCR extension step was added, with no apparent difference in the length distributions. To deplete total DNA samples of large fragments originating from insoluble chromatin prior to library preparation, samples were mixed with $1 / 2$ volume of HighPrep ${ }^{\text {TM }}$ PCR Clean-up System beads (MagBio Genomics cat. no. AC-60005), held 5-10 min, placed on a magnet stand, and the supernatant was collected. Based on the original sample volume, 0.8 volume of beads was added to the supernatant and held 5-10 min. Tubes were placed on a magnet stand, the supernatant discarded and the beads washed $1 \mathrm{x}$ with $80 \%$ ethanol prior to eluting in $15 \mathrm{uL} 10 \mathrm{mM}$ Tris- $\mathrm{HCl}$ pH 8. Library size distributions were resolved on an Agilent 4200 TapeStation.

\section{Data processing and analysis}

Barcoded libraries were mixed to achieve equimolar representation as desired aiming for a final concentration as recommended by the manufacturer for sequencing on an Illumina HiSeq 2500 2-lane Turbo flow cell. For each sample the following analysis steps were performed:

1) Aligned Illumina fastq files to a reference genome with bowtie 2 2.4.2 using parameters --end-to-end -very-sensitive --no-mixed --no-discordant --phred33 -I 10 -X 700

a) T19: GCF_000887095.1_ViralProj43573_genomic.fna from NCBI

Marseillevirus marseillevirus, strain T19 taxon 1559367

b) G648: G648_26-12-14_Genome_vD.fasta (Supplementary Information)

c) Widom 601 3x Array: Genome_601x3.fasta (Supplementary Information)

d) Drosophila melanogaster: dm6 from UCSC. Chronological list of D. melanogaster

ORFs: https://hgdownload.cse.ucsc.edu/goldenPath/dm6/database/refFlat.txt.gz 
2) Extracted aligned fragments from bowtie 2 sam files into bed files and divided into four fragment length subgroups: 1-, 1-100, 101-200, 201-300. Steps 3-8 were done for each of the four fragment lengths subgroups.

3) Computed percent GC for 10 base pairs on either end of the mapped fragments.

4) Made normalized count bedgraph files using bedtools 2.30.0 genomecov. Normalized counts are the fraction of counts at each base pair scaled by the size of the reference sequence so that if the scaled counts were uniformly distributed there would be 1 at each position.

5) Obtained annotations and selected $\geq 600$ bps long

a) T19: from NCBI: https://www.ncbi.nlm.nih.gov/nuccore/284504040

b) G648: Reference (1)

c) Drosophila melanogaster: dm6 annotation from UCSC: https://genome.ucsc.edu

6) Extracted normalized count (bedgraph) values for $600 \mathrm{bps}$ inside the selected genes from the TSS and from the TES using deepTools 3.3.1 computeMatrix with these parameters:

-S $<$ bigwig version of bedgraph file $>$-R genes.600 --referencePoint TSS -b 0 -a 600

-S $<$ bigwig version of bedgraph file $>$-R genes.600 --referencePoint TES -b 600 -a 0

7) Computed autocorrelation for of the extracted values for 1-300 lags using a custom program as R_lag= $1 /\left((\mathrm{n}-\mathrm{lag})^{*}\right.$ var $) * \operatorname{sum} \_1, \mathrm{n}-\mathrm{lag}\left(\mathrm{X} \_\mathrm{t}-\right.$ mean $)\left(\mathrm{X}_{-} \mathrm{t}+\mathrm{lag}-\mathrm{mean}\right)$ for each gene in each TSS and TES set.

8) Computed the mean and standard deviation of all 299 lags for each gene and reverse ranked the genes by standard deviation, selecting the four genes with the highest standard deviation for each TSS and TES set.

\section{References}

1. D. Sahmi-Bounsiar et al., Marseilleviruses: An Update in 2021. Front Microbiol 12, 648731 (2021).

2. M. Boyer et al., Giant Marseillevirus highlights the role of amoebae as a melting pot in emergence of chimeric microorganisms. Proc. Natl. Acad. Sci. U. S. A. 106, 21848 (2009).

3. A. J. Erives, Phylogenetic analysis of the core histone doublet and DNA topo II genes of Marseilleviridae: evidence of proto-eukaryotic provenance. Epigenetics Chromatin 10, 55 (2017).

4. M. I. Valencia-Sanchez et al., The structure of a virus-encoded nucleosome. Nat Struct Mol Biol 28, 413 (2021).

5. Y. Liu et al., Virus-encoded histone doublets are essential and form nucleosome-like structures. Cell 184, 4237 (2021).

6. A. Vannini, I. Marazzi, A small nucleosome from a weird virus with a fat genome. Mol. Cell 81, 3447 (2021).

7. Y. Liu, M. Krupovic, Genome chromatinization in giant double-stranded DNA viruses. Trends Biochem. Sci. 47, 3 (2022).

8. J. R. Schrad, J. S. Abrahao, J. R. Cortines, K. N. Parent, Structural and Proteomic Characterization of the Initiation of Giant Virus Infection. Cell 181, 1046 (2020).

9. Y. G. Kuznetsov et al., Atomic force microscopy investigation of the giant mimivirus. Virology 404, 127 (2010).

10. C. Xiao et al., Structural studies of the giant mimivirus. PLoS Biol 7, e92 (2009). 
11. N. Zauberman et al., Distinct DNA exit and packaging portals in the virus Acanthamoeba polyphaga mimivirus. PLoS Biol 6, e114 (2008).

12. M. Suzan-Monti, B. La Scola, L. Barrassi, L. Espinosa, D. Raoult, Ultrastructural characterization of the giant volcano-like virus factory of Acanthamoeba polyphaga Mimivirus. PLoS One 2, e328 (2007).

13. H. R. Chung et al., The effect of micrococcal nuclease digestion on nucleosome positioning data. PLoS One 5, e15754 (2010).

14. J. D. McGhee, G. Felsenfeld, Another potential artifact in the study of nucleosome phasing by chromatin digestion with micrococcal nuclease. Cell 32, 1205 (1983).

15. I. L. Cartwright, R. P. Hertzberg, P. B. Dervan, S. C. Elgin, Cleavage of chromatin with methidiumpropyl-EDTA . iron(II). Proc. Natl. Acad. Sci. U. S. A. 80, 3213 (1983).

16. H. Ishii, J. T. Kadonaga, B. Ren, MPE-seq, a new method for the genome-wide analysis of chromatin structure. Proc. Natl. Acad. Sci. U. S. A. 112, E3457 (2015).

17. G. P. Oliveira et al., The Investigation of Promoter Sequences of Marseilleviruses Highlights a Remarkable Abundance of the AAATATTT Motif in Intergenic Regions. J. Virol. 91, (2017).

18. I. Pagnier et al., A decade of improvements in Mimiviridae and Marseilleviridae isolation from amoeba. Intervirology 56, 354 (2013).

19. R. V. Chereji, T. D. Bryson, S. Henikoff, Quantitative MNase-seq accurately maps nucleosome occupancy levels. Genome Biol 20, 198 (2019).

20. S. K. Bhardwaj et al., Dinucleosome specificity and allosteric switch of the ISW1a ATP-dependent chromatin remodeler in transcription regulation. Nat Commun 11, 5913 (2020).

Acknowledgments: We thank Jim Kadonaga for MPE, Fred Hutch Genomics Shared Resource for sequencing services and Kami Ahmad for comments on the manuscript.

Funding: TDB, PBT and SH are supported by the Howard Hughes Medical Institute. PDI, MIV-S and KJA were supported by a grant from the David and Lucile Packard Foundation.

Author contributions: Conceptualization: K-JA and SH; Investigation: TDB, PDI, MIV-S, JGH, PBT, BLS, K-JA, SH; Writing - original draft: SH; Writing - review \& editing: TDB, PDI, MIV-S, JGH, PBT, K-JA, SH.

Competing interests: Authors declare that they have no competing interests. 

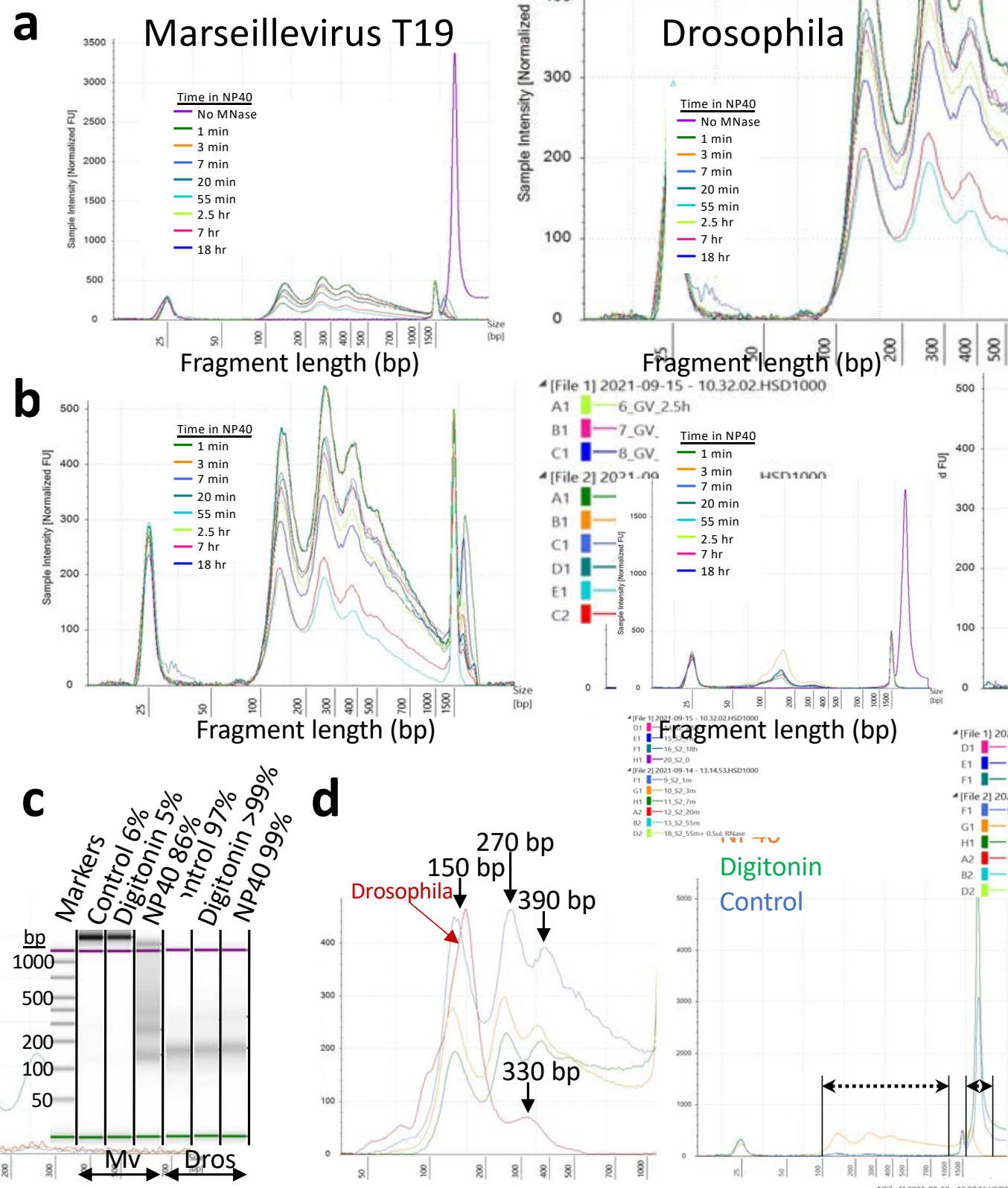

Figure S1: Permeabilization with NP-40 non-ionic detergent after capsid opening renders Marseillevirus chromatin immediately accessible to MNase digestion. a) Profile of Marseillevirus and Drosophila fragment lengths showing that NP40 completely permeabilizes $\mathrm{pH} 2$-treated and neutralized Marseillevirus particles to allow MNase digestion within 1 min. Estimated fragment sizes of peak midpoints are shown. b) Same as (a) showing that chromatin recovery is maximal in 1-3 minutes in NP40. c) Gel image of Marseillevirus (Mv) and Drosophila (Dros) chromatin extracted with no detergent (Control) or either Digitonin or NP-40 and digested with MNase at $10 \mathrm{U} /$ million cells for $5 \mathrm{~min}$ at $37^{\circ} \mathrm{C}$. d) Profiles of $\mathrm{Mv}$ and Dros fragment lengths showing recovery at 3 different concentrations. Estimated fragment sizes of peak midpoints are shown. e) Profiles of Mv lengths showing the ranges of signal used for calculating recovery: 100-1000 bp for MNase-released chromatin and >2 kb for undigested DNA. 


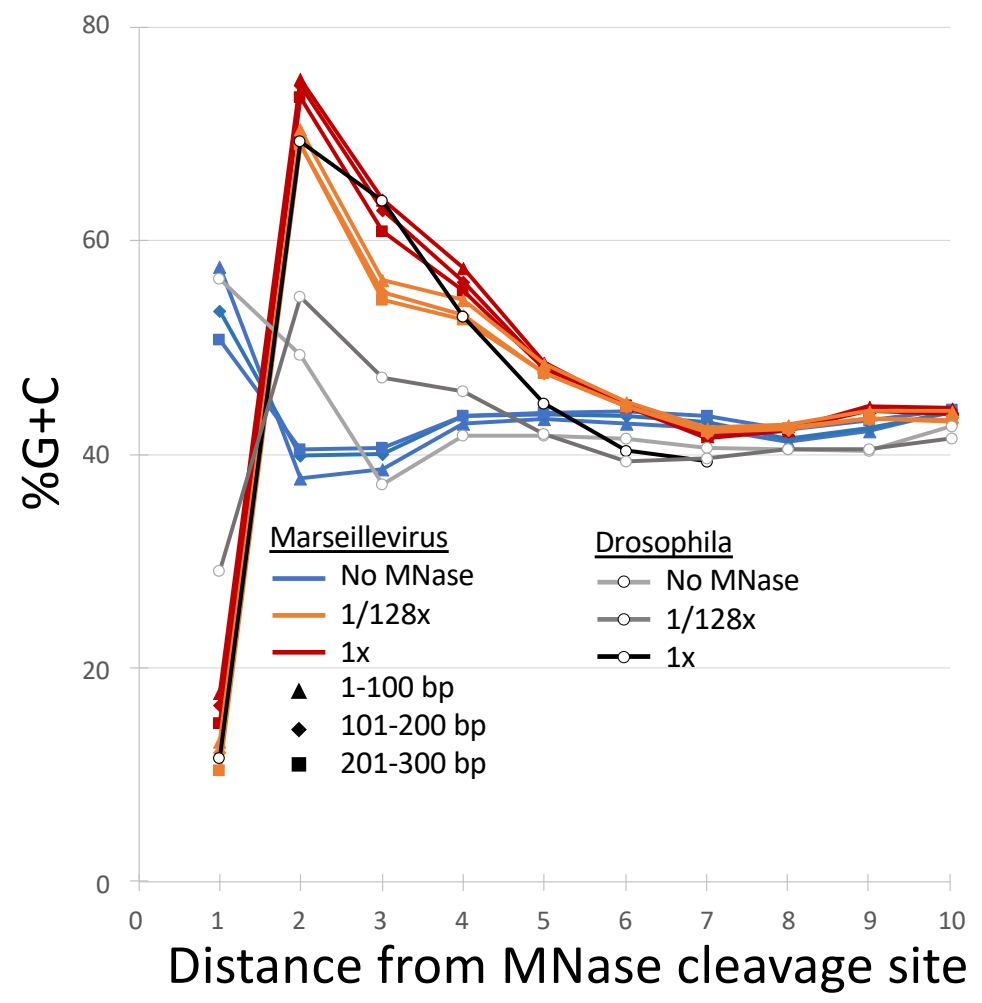

Figure S2: Preferential cleavage between $\mathrm{A} / \mathrm{T}$ base pairs in Marseillevirus and Drosophila. MNase is an endo-exonuclease that cleaves preferentially at A/T-rich DNA then 'nibbles' on ends until it reaches a G/C-rich 'clamp', as observed in our MNase-seq data for both Marseillevirus and Drosophila, whose genomes are both $44-45 \% \mathrm{G}+\mathrm{C}$ overall. 


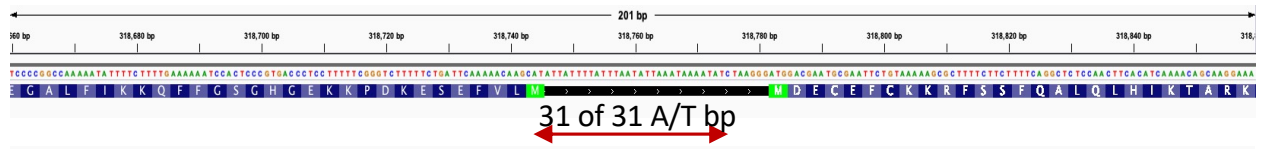

\section{No MNase}
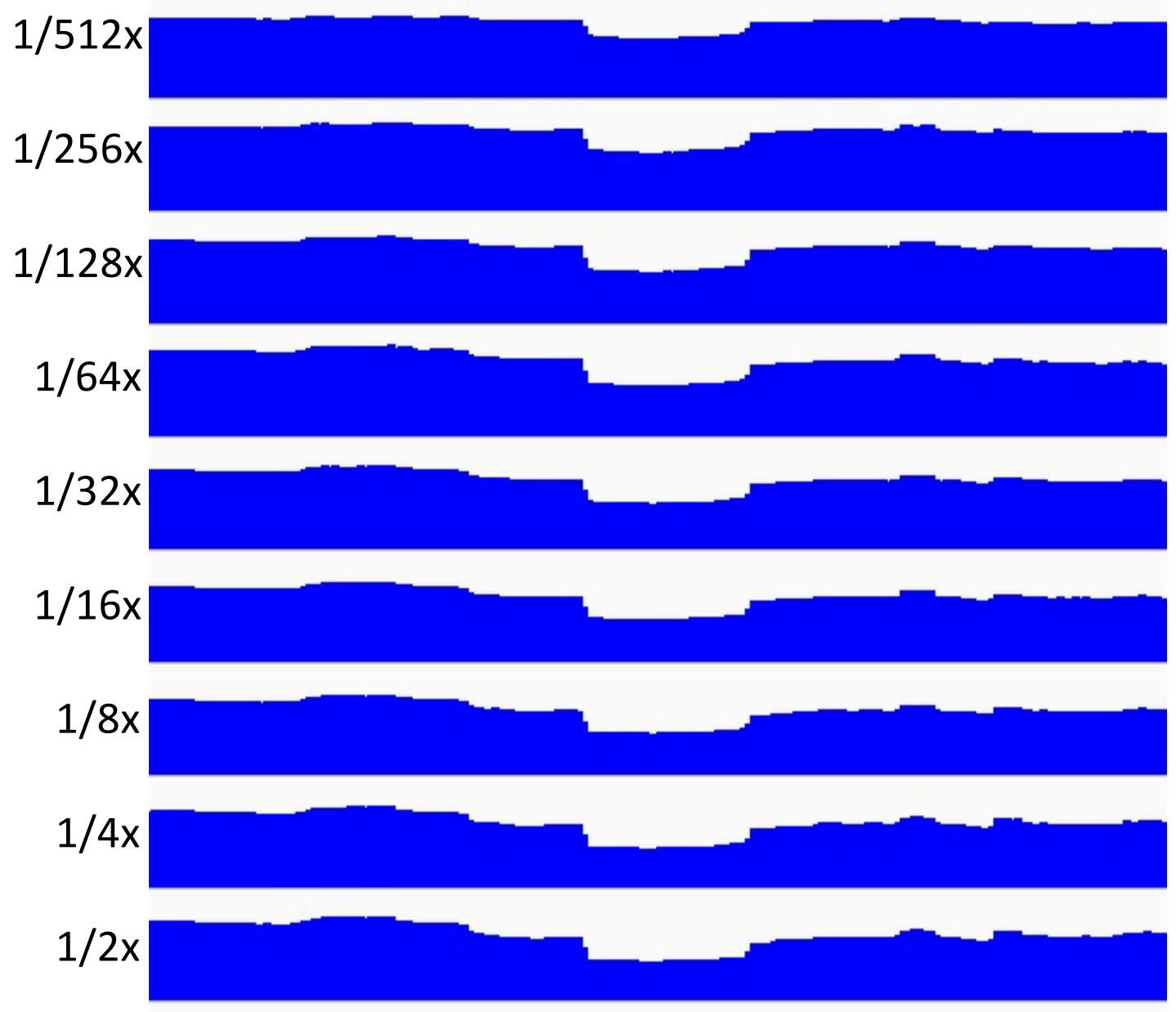

$1 x$

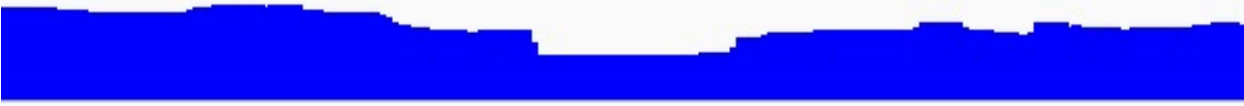

\section{MPE}

\section{Group autoscaled}

Figure S3: A/T-rich regions are preferentially digested by MNase. A Marseillevirus T19 bidirectional promoter with a $31 / 31 \mathrm{~A} / \mathrm{T}$ bp span is preferentially digested by MNase over a 500 -fold doubling series $(1 / 512 x, \ldots, 1 x)$, whereas MPE shows no preference. 

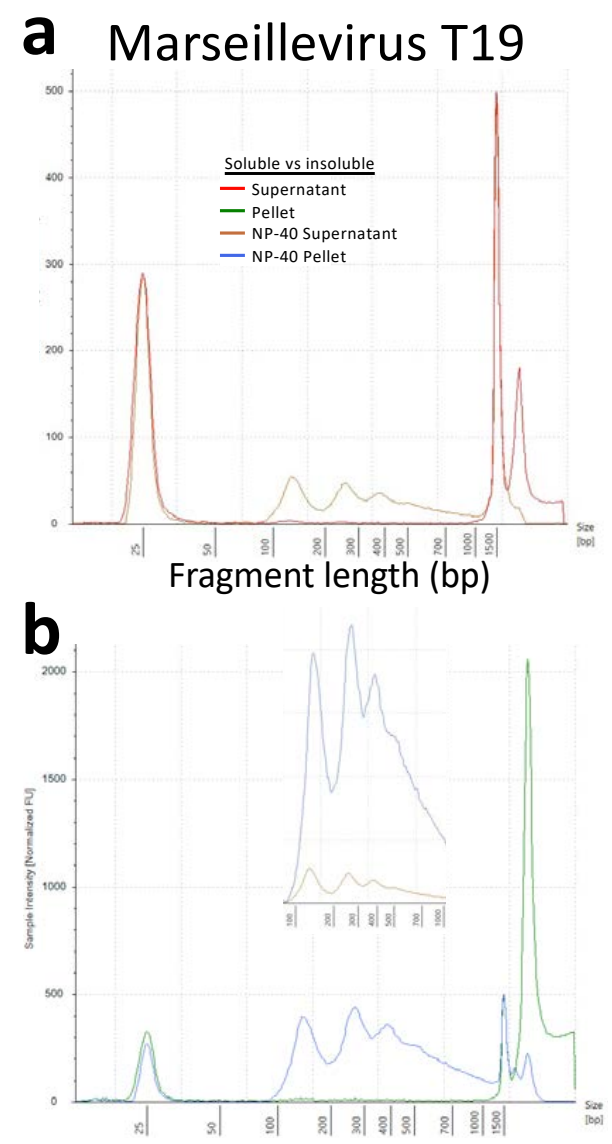

Fragment length (bp)

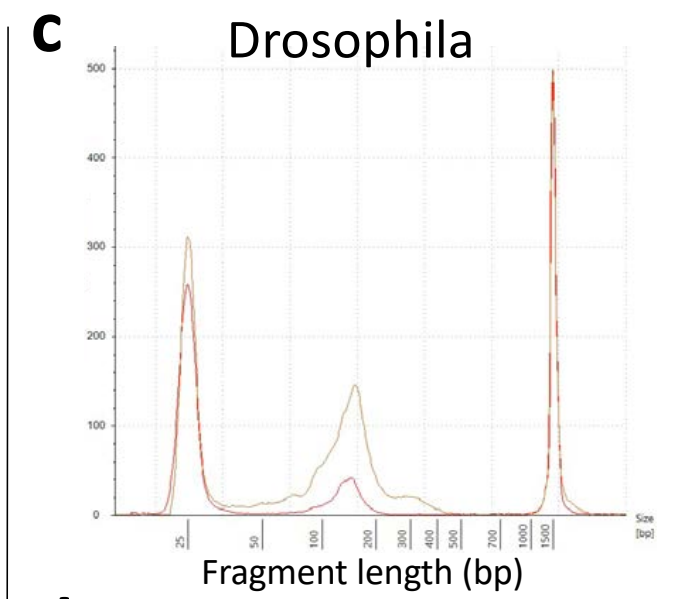

d

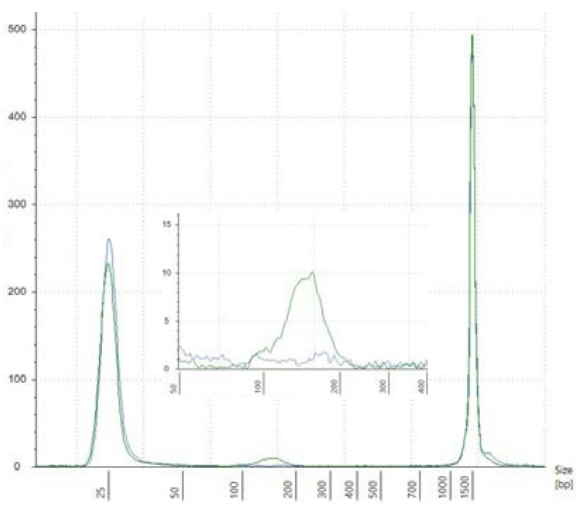

Fragment length (bp)

Figure S4: Marseillevirus nucleosomes are mostly insoluble after MNase treatment. Profiles of Marseillevirus T19 fragment lengths comparing \pm NP-40 in centrifugation to separate a) supernatant and b) pellet, where the inset shows that most of the NP-40-released and digested Marseillevirus chromatin is in the insoluble pellet fraction with similar nucleosome profiles in the two fractions. c-d) In contrast, most Drosophila chromatin is soluble whether or not treated with NP-40, where the inset shows a magnification of the pellet profile, which reveals quantitative release of eukaryotic chromatin into the supernatant with NP-40. 


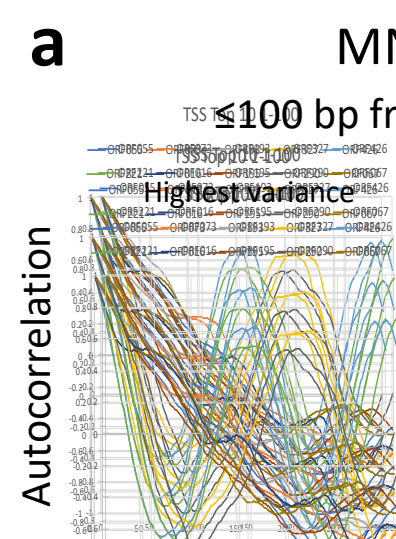

\section{MNase}

b

MPE

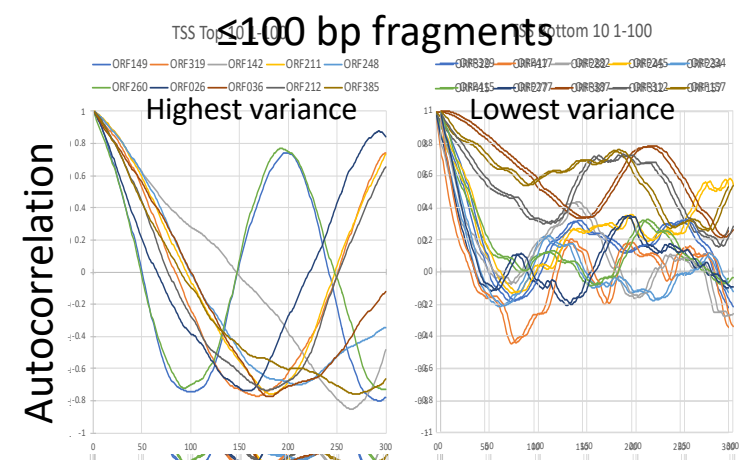

\section{1-200 bp fragments}
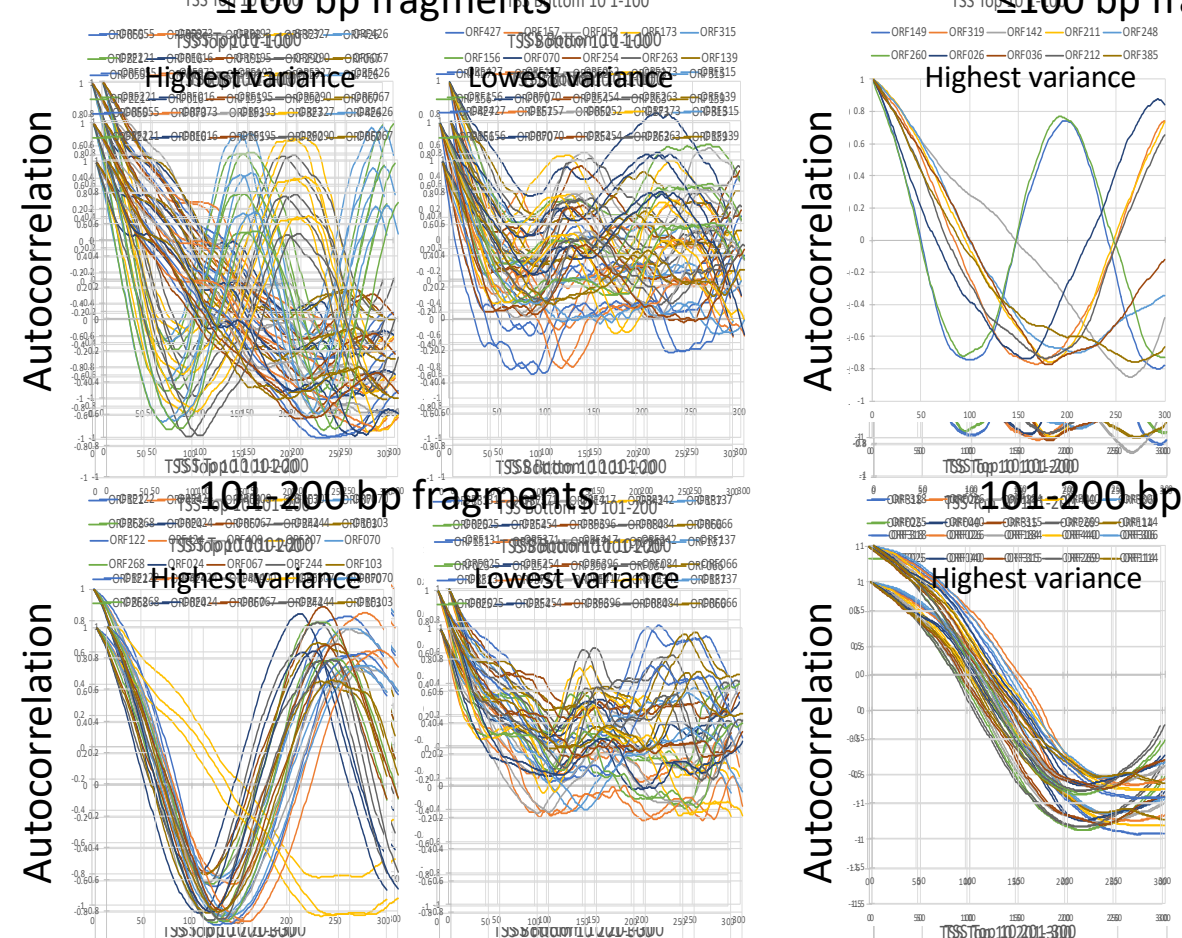

\section{1-300 bp fragments}
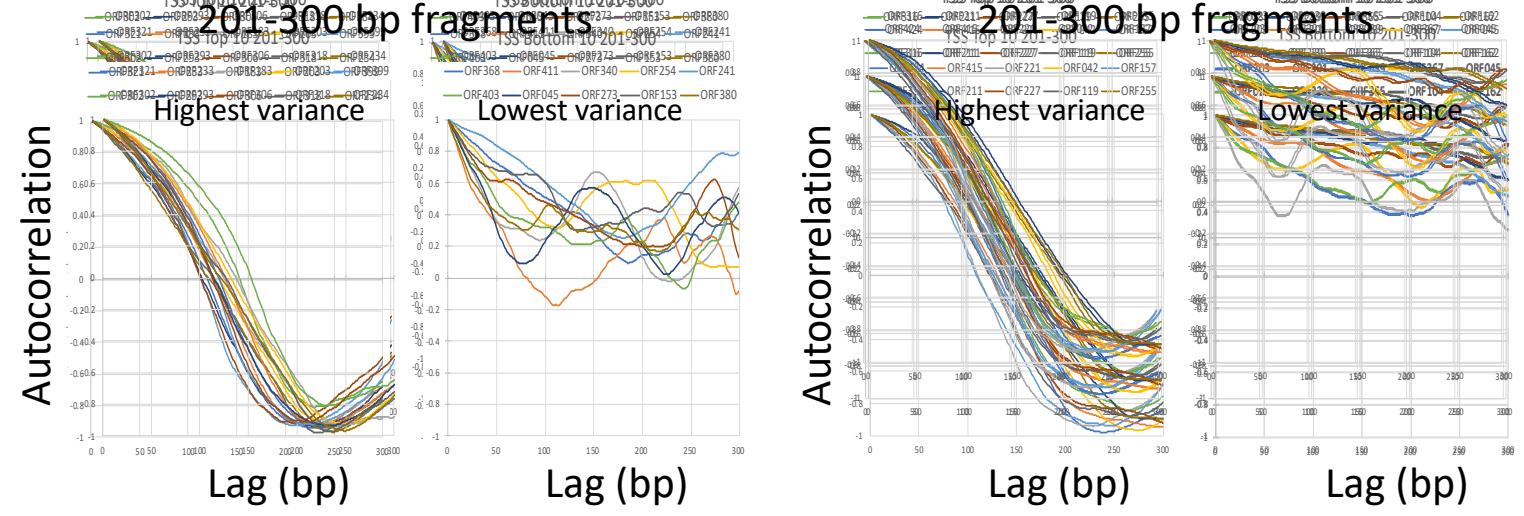

Figure S5: Autocorrelation illustrates periodicities over individual Marseillevirus gene bodies. To sensitively detect genes most likely to be phased, we plotted autocorrelations over the 600-bp span of each ORF for the 101-200 bp fragment size class using MNase-seq and MPE-seq data. Autocorrelations (-1 to +1) in 1-bp lag steps over a 5'-aligned 300-bp span are plotted for each of the 10 ORFs $\geq 600 \mathrm{bp}$ with the highest and lowest variance in amplitude for each size class for a) MNase-seq and $\mathbf{b}$ ) MPE-seq. 